\title{
THE ISOMETRIES OF SOME FUNCTION SPACES
}

KAREL DELEEUW, ${ }^{1}$ WALTER RUDIN ${ }^{2}$ AND JOHN WERMER ${ }^{3}$

I. Introduction. For each positive real number $p, H_{p}$ is the space of all functions which are analytic in the open unit disc and for which

$$
\|f\|_{p}=\sup _{0<r<1}\left[\frac{1}{2 \pi} \int_{-\pi}^{\pi}\left|f\left(r e^{i \theta}\right)\right|^{p} d \theta\right]^{1 / p}
$$

is finite. If $p \geqq 1, H_{p}$ is a complex Banach space, with (1.1) as norm.

The space of all bounded analytic functions in the open unit disc will be denoted by $H_{\infty}$. It also is a complex Banach space, with the norm

$$
\|f\|_{\infty}=\sup _{|z|<1}|f(z)| \text {. }
$$

$\mathrm{H}_{2}$ is a Hilbert space, and thus has many isometries; most of these do not preserve the additional structure which $H_{2}$ has as a consequence of the fact that it is a space of analytic functions. For $H_{1}$ and $H_{\infty}$, however, the opposite is true: here the isometries are induced by conformal maps of the unit disc. We shall prove the following:

THEOREM 1. Every linear isometry of $H_{\infty}$ onto $H_{\infty}$ is of the form

$$
(T f)(z)=\alpha \cdot f(t(z))
$$$$
\left(f \in H_{\infty},|z|<1\right) \text {, }
$$

where $\alpha$ is a complex number of absolute value 1 and $t$ is a conformal map of the unit disc onto itself.

Conversely, if $T$ satisfies (1.3), it is a linear isometry of $H_{\infty}$ onto $H_{\infty}$.

TheOREM 2. Every linear isometry of $H_{1}$ onto $H_{1}$ is of the form

$$
(T f)(z)=\alpha \cdot t^{\prime}(z) \cdot f(t(z)),
$$$$
\left(f \in H_{1} ;|z|<1\right),
$$

where $\alpha$ and $t$ are as in Theorem 1. Conversely, if $T$ satisfies (1.4), it is a linear isometry of $H_{1}$ onto $H_{1}$.

These theorems fail if $T$ is not assumed to be onto, since multiplication by any Blaschke product is a linear isometry of $H_{p}$ into $H_{p}$ $(1 \leqq p \leqq \infty)$. Also, Halsey Royden has pointed out that one cannot replace "linear" by "real-linear" in the hypotheses; for if $\tilde{f}(z)$ is the

Received by the editors January 15, 1960.

1 Supported by the Air Force Office of Scientific Research.

2 Research Fellow of the Alfred P. Sloan Foundation.

'Supported by National Science Foundation grant G-5866. 
complex conjugate of $f(\bar{z})$, then the map $f \rightarrow \bar{f}$ is a real-linear isometry of $H_{p}$ onto $H_{p}(1 \leqq p \leqq \infty)$.

Before we turn to the proofs of these results, we consider a more abstract situation.

II. Isometries of sup-norm algebras. A semi-simple commutative Banach algebra $A$ with unit whose Gelfand representation is a uniformly closed subalgebra of $C(\Delta)$ (the space of all continuous functions on the maximal ideal space $\Delta$ of $A$ ) will be called a sup-norm algebra. We shall regard any such algebra as a uniformly closed subalgebra of $C(\Delta)$, equipped with the norm

$$
\|f\|=\sup _{x \in \Delta}|f(x)| \quad(f \in A) .
$$

TheOREM 3. Every linear isometry of a sup-norm algebra $A$ onto itself is of the form

$$
T f=\alpha \cdot T_{1} f \quad(f \in A),
$$

where $\alpha \in A, \alpha^{-1} \in A,|\alpha(x)|=1$ for all $x \in \Delta$, and $T_{1}$ is an automorphism of $A$.

Proof. To begin with, we regard $A$ and $C(\Delta)$ merely as Banach spaces, and we let $S\left(A^{*}\right)$ and $S\left(C^{*}\right)$ denote the unit balls of their dual spaces $A^{*}$ and $C^{*}$. If $Y$ is the subspace of $C^{*}$ which annihilates $A$, then there is a homomorphism $h$ of $C^{*}$ onto $A^{*}$ whose kernel is $Y$. If $L$ is an extreme point of $S\left(A^{*}\right)$, then $h^{-1}(L) \cap S\left(C^{*}\right)$ is nonempty (by the Hahn-Banach theorem), weak*-compact and convex and hence has an extreme point, by the Krein-Milman theorem; any such extreme point is also an extreme point of $S\left(C^{*}\right)$, and hence is of the form $\lambda L_{x}$, with $|\lambda|=1$. (We write $L_{x}$ for the functional which assigns to each $f$ the number $f(x)$.) We have proved:

To every extreme point $L$ of $S\left(A^{*}\right)$ there corresponds a complex number $\lambda$ of absolute value 1 and a point $x \in \Delta$ such that

$$
L f=\lambda f(x)
$$

Furthermore, $\lambda$ and $x$ are uniquely determined by $L$, since $A$ separates points on $\Delta$.

Let $\partial A$ be the set of all $x$ obtained in this way. We note that $A$ has the maximum modulus property with respect to $\partial A$ :

To every $f \in A$ there corresponds a point $x \in \partial A$ such that $\|f\|=|f(x)|$. Indeed, $\|f\|=\max |L f|\left(L \in S\left(A^{*}\right)\right)$; the maximum is attained, since $S\left(A^{*}\right)$ is weak ${ }^{*}$-compact, and it is attained at an extreme point of $S\left(A^{*}\right)$. 
The preceding considerations were suggested by Lemmas 4.3 and 6.1 of $[1]$.

Now let $T$ be a linear isometry of $A$ onto $A$. Its adjoint $T^{*}$ is an isometry of $A^{*}$ onto $A^{*}$, and thus carries the set of extreme points of $S\left(A^{*}\right)$ onto itself. Hence to each $x \in \partial A$ there corresponds a complex number $\alpha(x),|\alpha(x)|=1$, and a point $\phi(x) \in \partial A$, such that

$$
T^{*} L_{x}=\alpha(x) L_{\phi}(x) \text {. }
$$

That is to say,

$$
(T f)(x)=L_{x} T f=T^{*} L_{x} f=\alpha(x) L_{\phi(x)} f
$$

or

$$
(T f)(x)=\alpha(x) f(\phi(x)) \quad(f \in A, x \in \partial A) .
$$

Taking $f=1$ in (2.6), we see that $\alpha$ coincides with $T 1$ on $\partial A$. We extend the definition of $\alpha$ to $\Delta$ by setting $\alpha(x)=(T 1)(x)$ for all $x \in \Delta$. Then $\alpha \in A$.

So far we have only used the linear structure of $A$. If we now substitute a product $f g$ into (2.6), we obtain

$$
(T(f g))(x)=\alpha(x) f(\phi(x)) g(\phi(x)) .
$$

Multiplying by $\alpha(x)$, this becomes

$$
\alpha(x)(T(f g))(x)=(T f)(x) \cdot(T g)(x) \quad(f, g \in A ; x \in \partial A),
$$

by (2.6). Thus the function $\alpha \cdot T(f g)-(T f) \cdot(T g)$, which belongs to $A$, is 0 on $\partial A$, and hence is identically 0 , by the maximum modulus property established above. Thus

$$
\alpha \cdot T(f g)=(T f) \cdot(T g) \quad(f, g \in A) .
$$

Choosing $f$ and $g$ so that $T f=T g=1$ (which is possible, since $T$ is onto), (2.9) shows that $\alpha^{-1} \in A$. The maximum modulus property, applied to $\alpha$ and to $\alpha^{-1}$, then implies that $|\alpha(x)|=1$ for all $x \in \Delta$.

Finally, put $T_{1} f=\alpha^{-1} T f$. Then $T_{1}$ is a one-to-one linear map of $A$ onto $A$ which is also multiplicative, by (2.9), and this completes the proof.

III. Proofs of Theorems 1 and 2. $H_{\infty}$ is a sup-norm algebra, as follows readily from the definition (1.2) of the norm. Its maximal ideal space $\Delta$ has a complicated structure, but all we need to know here is that $\Delta$ contains the open unit disc. Every automorphism of $H_{\infty}$ is induced by a conformal map of the unit disc $[3 ; 4$, Theorem 9], and the constants are the only functions in $H_{\infty}$ whose absolute value is 
constant. The direct part of Theorem 1 is thus a consequence of Theorem 3; the converse is trivial.

We note, in passing, that the same proof applies if $H_{\infty}$ is replaced by the ring of all single-valued bounded analytic functions in a domain which is "maximal" in the sense in which this term was defined in [4].

Our proof of Theorem 2 leans heavily on some of the results obtained in [2], and we refer to that paper for the notion of "outer function" and for those properties of $H_{1}$ which we shall use.

In [2] it is proved that the extreme points of the unit ball of $H_{1}$ are precisely the outer functions of norm 1 , and that the closure of this set of extreme points consists of all $f \in H_{1}$ with $\|f\|_{1}=1$ which have no zero in $|z|<1$.

If now $T$ is an isometry of $H_{1}$ onto $H_{1}$, it follows that $f$ has no zero in $|z|<1$ if and only if $T f$ has no zero either. If $k=T 1$ and if $\lambda$ is a complex number, the linearity of $T$ shows that $T(f-\lambda)=T f-\lambda k$. Since $f-\lambda$ has no zero if and only if $T f-\lambda k$ has no zero, it follows that $\lambda$ is in the range of $f$ if and only if $\lambda$ is in the range of $k^{-1} T f$. We have proved:

For every $f \in H_{1}, f$ and $k^{-1} T f$ have the same range.

We set $U f=k^{-1} T f$. The restriction of $U$ to $H_{\infty}$ is then a linear isometry of $H_{\infty}$ into $H_{\infty}$. Moreover, if $b \in H_{\infty}$ and $f=T^{-1}(b k)$, then $f \in H^{1}$, and

$$
U f=k^{-1} T f=b ;
$$

thus $k^{-1} T f \in H_{\infty}$, and since $f$ has the same range, $f \in H_{\infty}$. We have proved that $U$ maps $H_{\infty}$ onto $H_{\infty}$. Theorem 1 applies to $U$ and yields

$$
(T f)(z)=k(z) f(t(z)) \quad\left(f \in H_{\infty},|z|<1\right),
$$

since $U 1=1$; here $t$ is a conformal map of the unit disc onto itself. Next,

$$
\|T f\|_{1}=\frac{1}{2 \pi} \int_{-\pi}^{\pi}\left|k\left(e^{i \theta}\right)\right| \cdot\left|f\left(t\left(e^{i \theta}\right)\right)\right| d \theta
$$

for every $f \in H_{\infty}$, and

$$
\|f\|_{1}=\frac{1}{2 \pi} \int_{-\pi}^{\pi}\left|f\left(e^{i \theta}\right)\right| d \theta=\frac{1}{2 \pi} \int_{-\pi}^{\pi}\left|t^{\prime}\left(e^{i \theta}\right)\right| \cdot\left|f\left(t\left(e^{i \theta}\right)\right)\right| d \theta .
$$

To every bounded measurable real function $u$ on $|z|=1$ there corresponds an $f \in H_{\infty}$ such that $|f|=e^{u}$ a.e. on $|z|=1$. Since $T$ is an isometry of $H_{1}$, the last two terms of (3.3) and (3.4) are equal, for all $f \in H_{\infty}$, so that 


$$
\int_{-\pi}^{\pi}|k| e^{u} d \theta=\int_{-\pi}^{\pi}\left|t^{\prime}\right| e^{u} d \theta
$$

for every bounded measurable real $u$. This is possible only if $|k|$ $=\left|t^{\prime}\right|$ a.e. on $|z|=1$. Since both $k$ and $t^{\prime}$ are outer functions $(k=T 1$, and 1 is outer!), it follows that $k=\alpha t^{\prime}$, with $|\alpha|=1$.

Thus (3.2) becomes (1.4), valid for all $f \in H_{\infty}$. Since $H_{\infty}$ is dense in $H_{1}$, we see that (1.4) holds for all $f \in H_{1}$, and the direct part of Theorem 2 is proved. The converse is trivial.

ADDED IN PROOF. After completion of this paper, we noticed a paper by M. Nagasawa, Isomorphisms between commutative Banach algebras with an application to rings of analytic functions, Kōdai Math. Sem. Rep. vol. 11 (1959) pp. 182-188. Using the fact that sup-norm algebras may be regarded as operator algebras on a Hilbert space, Nagasawa obtains our Theorems 1 and 2 .

\section{REFERENCES}

1. Errett Bishop and Karel deLeeuw, The representations of linear functionals by measures on sets of extreme points, Ann. Inst. Fourier, Grenoble (to appear).

2. Karel deLeeuw and Walter Rudin, Extreme points and extremum problems in $H_{1}$, Pacific J. Math. vol. 8 (1958) pp. 467-485.

3. Shizuo Kakutani, Rings of analytic functions, Lectures on functions of a complex variable, Ann Arbor, 1955.

4. Walter Rudin, Some theorems on bounded analytic functions, Trans. Amer. Math. Soc. vol. 78 (1955) pp. 333-342.

STANFORD UNIVERSITY,

UNIVERSITY OF ROCHESTER, AND

BROWN UNIVERSITY 